\title{
Complete Periodic Synchronization of Memristor-Based Neural Networks with Time-Varying Delays
}

\author{
Huaiqin Wu, Luying Zhang, Sanbo Ding, Xueqing Guo, and Lingling Wang \\ Department of Applied Mathematics, Yanshan University, Qinhuangdao 066001, China \\ Correspondence should be addressed to Huaiqin Wu; huaiqinwu@ysu.edu.cn
}

Received 6 April 2013; Revised 4 June 2013; Accepted 8 June 2013

Academic Editor: Zhengqiu Zhang

Copyright ( 2013 Huaiqin Wu et al. This is an open access article distributed under the Creative Commons Attribution License, which permits unrestricted use, distribution, and reproduction in any medium, provided the original work is properly cited.

\begin{abstract}
This paper investigates the complete periodic synchronization of memristor-based neural networks with time-varying delays. Firstly, under the framework of Filippov solutions, by using $M$-matrix theory and the Mawhin-like coincidence theorem in setvalued analysis, the existence of the periodic solution for the network system is proved. Secondly, complete periodic synchronization is considered for memristor-based neural networks. According to the state-dependent switching feature of the memristor, the error system is divided into four cases. Adaptive controller is designed such that the considered model can realize global asymptotical synchronization. Finally, an illustrative example is given to demonstrate the validity of the theoretical results.
\end{abstract}

\section{Introduction}

Memristor, as the fourth fundamental passive circuit, was firstly postulated by Chua [1] in 1971. On May 1, 2008, the Hewlett-Packard (HP) research team announced their realization of a memristor prototype, with an official publication in Nature $[2,3]$. This new circuit element of memristor shares many properties of resistors and shares the same unit of measurement. Recently, memristor has received a great deal of attention because of its potential applications in next generation computer and powerful brain-like "neural" computer. The papers [4-21] have given a detailed introduction on the memristor, so readers can consult [4-21] to get more explanation. As noted in [10], from a systems-theoretic point of view and a mathematical point of view, memristor dynamics strictly obey Bernoulli's nonlinear differential equation, so the mathematical framework and its usefulness are worth studying. The paper [10] by $\mathrm{Wu}$ and Zeng discussed the exponential stabilization of memristive neural networks with time delays. The papers [11-14] investigated the synchronization and antisynchronization control of a class of memristor-based recurrent neural networks. A series of results on stability analysis of memristor-based recurrent neural networks were presented in [15-18]. The papers [1921] dealt with the existence and stability of periodic solution of almost periodic of a class of memristor-based recurrent neural networks.

Different from the previous works, in this paper, we will study complete periodic synchronization of memristorbased neural networks described by the following differential equation:

$$
\begin{array}{r}
\dot{x}_{i}(t)=-d_{i}\left(x_{i}(t)\right) x_{i}(t)+\sum_{j=1}^{n} a_{i j}\left(x_{i}(t)\right) f_{j}\left(x_{j}(t)\right) \\
+\sum_{j=1}^{n} \mathrm{~b}_{i j}\left(x_{i}(t)\right) g_{j}\left(x_{j}\left(t-\tau_{i j}(t)\right)\right)+I_{i}(t), \\
t \geq 0, i=1,2, \ldots, n,
\end{array}
$$

where

$$
\begin{aligned}
& d_{i}\left(x_{i}(t)\right)= \begin{cases}\dot{d}_{i}, & \left|x_{i}(t)\right| \leq T_{i}, \\
\dot{d}_{i}, & \left|x_{i}(t)\right|>T_{i},\end{cases} \\
& a_{i j}\left(x_{i}(t)\right)= \begin{cases}\dot{a}_{i j}, & \left|x_{i}(t)\right| \leq T_{i}, \\
\dot{a}_{i j}, & \left|x_{i}(t)\right|>T_{i},\end{cases} \\
& b_{i j}\left(x_{i}(t)\right)= \begin{cases}\hat{b}_{i j}, & \left|x_{i}(t)\right| \leq T_{i}, \\
\dot{b}_{i j}, & \left|x_{i}(t)\right|>T_{i} .\end{cases}
\end{aligned}
$$


in which switching jumps $T_{i}>0, \dot{d}_{i}>0, \grave{d}_{i}>0, \dot{a}_{i j}, \grave{a}_{i j}$, $\dot{b}_{i j}, \grave{b}_{i j}, i, j=1,2, \ldots, n$, are constants; $f_{j}$ and $g_{j}$ are feedback functions, $\tau_{i j}(t)$ is the time delay with $0 \leq \tau_{i j}(t) \leq \tau$ and $\dot{\tau}_{i j} \leq \mu_{i j}<1$ ( $\tau$ and $\mu_{i j}$ are negative constants). At first glance, one might intuitively believe that the chaotic motion is more complicated compared with the periodic motion, the synchronization of chaotic oscillators is also complicated than those of periodic oscillators [22]. However, this is not always true, just as indicated in $[23,24]$, where an opposite result was given.

The rest of this paper is organized as follows. In Section 2, some preliminaries are introduced. In Section 3, the proof of the existence of periodic solutions is presented. Complete periodic synchronization is discussed in Section 4. In Section 5, a numerical example is presented to demonstrate the validity of the proposed results. Some conclusions are drawn in Section 6.

Notation. $\mathbb{R}$ denotes the set of real numbers, $\mathbb{R}^{n}$ denotes the $n$-dimensional Euclidean space, and $\mathbb{R}^{m \times n}$ denotes the set of all $m \times n$ real matrices. Given the vectors $x=\left(x_{1}, \ldots, x_{n}\right)^{T}$, $y=\left(y_{1}, \ldots, y_{n}\right)^{T} \in \mathbb{R}^{n},\|x\|=\left(\sum_{i=1}^{n} x_{i}^{2}\right)^{1 / 2}, x^{T} y=$ $\sum_{i=1}^{n} x_{i} y_{i}$. For $r>0, C\left([-r, 0] ; \mathbb{R}^{n}\right)$ denotes the family of continuous function $\varphi$ from $[-r, 0]$ to $\mathbb{R}^{n}$ with the norm $\|\varphi\|=\sup _{-r \leq s \leq 0}|\varphi(s)| .[\cdot, \cdot]$ represents the interval. $\operatorname{co}(Q)$ denotes the closure of the convex hull of $Q$. $E_{n}$ denotes the identity matrix of size $n$. A vector or matrix $A \geq 0$ means that all entries of $A$ are greater than or equal to zero; $A>0$ can be defined similarly. For vectors or matrices $A$ and $B, A \geq B$ (or $A>B$ ) means that $A-B \geq 0$ (or $A-B>0) . K\left(\mathbb{R}^{n}\right)$ denotes the collection of all nonempty compact subsets of $\mathbb{R}$ with the Hausdorff metric $\rho$ defined by $\rho(A, B)=\max \{\beta(A, B), \beta(B, A)\}, A, B \in K\left(\mathbb{R}^{n}\right)$, and $\rho(A, B)=\sup \{\operatorname{dist}(x, B): x \in A\}, \rho(B, A)=\sup \{\operatorname{dist}(y, A):$ $y \in B\}, K v\left(\mathbb{R}^{n}\right)=\left\{A \in K\left(\mathbb{R}^{n}\right): A\right.$ is convex $\}$.

\section{Preliminaries}

In this section, we give some definitions and properties, which are needed later.

Definition 1 (see [25]). Suppose $E \subseteq \mathbb{R}^{n}$; then $x \rightarrow F(x)$ is called a set-valued map from $E \rightarrow \mathbb{R}^{n}$, if for each point $x \in$ $E$, there exists a nonempty set $F(x) \subseteq \mathbb{R}^{n}$. A set-valued map $F$ with nonempty values is said to be upper semicontinuous (USC) at $x_{0} \in E$, if for any open set $N$ containing $F\left(x_{0}\right)$, there exists a neighborhood $M$ of $x_{0}$ such that $F(M) \subseteq N$. The map $F(x)$ is said to have a closed (convex, compact) image if for each $x \in E, F(x)$ is closed (convex, compact).

Definition 2 (see [26]). For the system $\dot{x}(t)=f(t, x), x \in$ $\mathbb{R}^{n}$, with discontinuous right-hand sides, a set-valued map is defined as

$$
F(t, x)=\bigcap_{\delta>0} \bigcap_{\mu(N)=0} \operatorname{co}[f(B(x, \delta) \backslash N)],
$$

where $\operatorname{co}[E]$ is the closure of the convex hull of set $E, B(x, \delta)=$ $\{y:\|y-x\| \leq \delta\}$ and $\mu(N)$ is Lebesgue measure of set
$N$. A solution in Filippov's sense of the Cauchy problem for this system with initial condition $x(0)=x_{0}$ is an absolutely continuous function $x(t), t \in[0, T]$, which satisfies $x(0)=x_{0}$ and differential inclusion

$$
\dot{x}(t) \in F(t, x), \quad \text { for a.a } t \in[0, T] .
$$

The initial value associated with system (1) is $x_{i}(t)=$ $\phi(t) \in C([-\tau, 0] ; \mathbb{R}), i=1,2, \ldots, n$. Let $\underline{d}_{i}=\min \left\{\dot{d}_{i}, \grave{d}_{i}\right\}$, $\bar{d}_{i}=\max \left\{\hat{d}_{i}, \grave{d}_{i}\right\}, \underline{a}_{i j}=\min \left\{\hat{a}_{i j}, \grave{a}_{i j}\right\}, \bar{a}_{i j}=\max \left\{\dot{a}_{i j}, \grave{a}_{i j}\right\}$, $\underline{b}_{i j}=\min \left\{\hat{b}_{i j}, \grave{b}_{i j}\right\}, \bar{b}_{i j}=\max \left\{\hat{b}_{i j}, \grave{b}_{i j}\right\}, \tilde{a}_{i j}=\max \left\{\left|\underline{a}_{i j}\right|,\left|\bar{a}_{i j}\right|\right\}$, and $\widetilde{b}_{i j}=\max \left\{\left|\underline{b}_{i j}\right|,\left|\bar{b}_{i j}\right|\right\}$. We define

$$
\begin{array}{r}
\operatorname{co}\left(d_{i}\left(x_{i}(t)\right)\right)= \begin{cases}\dot{d}_{i}, & \left|x_{i}(t)\right|<T_{i}, \\
{\left[\underline{d}_{i}, \bar{d}_{i}\right],} & \left|x_{i}(t)\right|=T_{i}, \\
\dot{d}_{i}, & \left|x_{i}(t)\right|>T_{i},\end{cases} \\
\operatorname{co}\left(a_{i j}\left(x_{i}(t)\right)\right)= \begin{cases}\dot{a}_{i j}, & \left|x_{i}(t)\right|<T_{i}, \\
{\left[\underline{a}_{i j}, \bar{a}_{i j}\right],} & \left|x_{i}(t)\right|=T_{i}, \\
\dot{a}_{i j}, & \left|x_{i}(t)\right|>T_{i},\end{cases} \\
\operatorname{co}\left(b_{i j}\left(x_{i}(t)\right)\right)= \begin{cases}\hat{b}_{i j}, & \left|x_{i}(t)\right|<T_{i}, \\
{\left[\underline{b}_{i j}, \bar{b}_{i j}\right],} & \left|x_{i}(t)\right|=T_{i}, \\
\dot{b}_{i j}, & \left|x_{i}(t)\right|>T_{i},\end{cases}
\end{array}
$$

Clearly, system (1) is a differential equation with discontinuous right-hand side; its solution in the conventional sense does not exist. Inspired by $[10,20,21,27-30]$, we adopt the following definition of the solution in the sense of Filippov for system (1).

Definition 3. Suppose that $\phi(s)=\left(\phi_{1}(s), \phi_{2}(s), \ldots, \phi_{n}(s)\right)^{T} \in$ $C\left([-\tau, 0] ; \mathbb{R}^{n}\right)$ is a continuous function. An absolutely continuous function $x(t)$ is said to be a solution with initial data $\phi(s)$ of system (1), if $x(t)$ satisfies the differential inclusion

$$
\begin{aligned}
\dot{x}_{i}(t) \in & -\operatorname{co}\left(d_{i}\left(x_{i}(t)\right)\right) x_{i}(t) \\
& +\sum_{j=1}^{n} \operatorname{co}\left(a_{i j}\left(x_{i}(t)\right)\right) f_{j}\left(x_{j}(t)\right) \\
& +\sum_{j=1}^{n} \operatorname{co}\left(b_{i j}\left(x_{i}(t)\right)\right) g_{j}\left(x_{j}\left(t-\tau_{i j}(t)\right)\right)+I_{i}(t),
\end{aligned}
$$

for all $i=1,2, \ldots, n$, or equivalently, there exist $d_{i}(t) \in$ $\operatorname{co}\left(d_{i}\left(x_{i}(t)\right)\right), a_{i j}(t) \in \operatorname{co}\left(a_{i j}\left(x_{i}(t)\right)\right)$, and $b_{i j}(t) \in \operatorname{co}\left(b_{i j}\left(x_{i}(t)\right)\right)$, such that

$$
\begin{aligned}
\dot{x}_{i}(t)= & -d_{i}(t) x_{i}(t)+\sum_{j=1}^{n} a_{i j}(t) f_{j}\left(x_{j}(t)\right) \\
& +\sum_{j=1}^{n} b_{i j}(t) g_{j}\left(x_{j}\left(t-\tau_{i j}(t)\right)\right)+I_{i}(t) .
\end{aligned}
$$

Remark 4. From the theoretical point of view, the above parameters $d_{i}(t), a_{i j}(t)$, and $b_{i j}(t)$ in (7) are measurable functions and depend on the state $x_{i}(t)$ and time $t$. 
Definition 5. We say that real matrix $M=\left(m_{i j}\right) \in \mathbb{R}^{n \times n}$ is an $M$-matrix, if and only if we have $m_{i j} \leq 0, i, j=1,2, \ldots, n$, $i \neq j$, and all successive principal minors of $M$ are positive.

Lemma 6 (see [31]). Let $M$ be an $n \times n$ matrix with nonpositive off-diagonal elements. Then $M$ is an $M$-matrix if and only if one of the following conditions hold:

(1) there exists a vector $\xi=\left(\xi_{1}, \xi_{2}, \ldots, \xi_{n}\right)>(0,0, \ldots, 0)$ such that $\xi M>0$;

(2) there exists a vector $\eta=\left(\eta_{1}, \eta_{2}, \ldots, \eta_{n}\right)^{T}>(0,0, \ldots$, $0)^{T}$ such that $M \eta>0$.

Lemma 7 (Mawhin-Like Coincidence Theorem [32]). Suppose that $F: \mathbb{R} \times \mathbb{R}^{n} \rightarrow K v\left(\mathbb{R}^{n}\right)$ is USC and $\omega$-periodic in $t$. If the following conditions hold:

(1) there exists a bounded open set $\Omega \subseteq C_{\omega}$, the set of all continuous, $\omega$-periodic functions: $\mathbb{R} \rightarrow \mathbb{R}^{n}$, such that for any $\lambda \in(0,1)$, each $\omega$-periodic function $x(t)$ of the inclusion

$$
\dot{x}(t) \in \lambda F(t, x)
$$

satisfies $x \notin \partial \Omega$ if it exists;

(2) each solution $u \in \mathbb{R}^{n}$ of the inclusion

$$
0 \in \frac{1}{\omega} \int_{0}^{\omega} F(t, u) d t=\omega(u)
$$

satisfies $u \notin \partial \Omega \bigcap \mathbb{R}^{n}$;

(3) $\operatorname{deg}\left(\omega, \Omega \bigcap \mathbb{R}^{n}, 0\right) \neq 0$,

then differential inclusion (4) has at least one $\omega$ periodic solution $x(t)$ with $x \in \bar{\Omega}$.

To proceed with our analysis, we need the following assumptions for system (1).

$\left(\mathrm{A}_{1}\right) I_{i}(t)$ and $\tau_{i j}(t)$ are continuous $\omega$-periodic functions.

$\left(\mathrm{A}_{2}\right)$ For $i=1,2, \ldots, n$, for all $s_{1}, s_{2} \in \mathbb{R}, s_{1} \neq s_{2}$, the neural activation function $g_{i}$ is bounded and $f_{i}, g_{i}$ satisfy Lipschitz condition; that is, there exist $\varrho_{i}>0, \rho_{i}>0$ and $G_{i}$, such that

$$
\begin{gathered}
\left|f_{i}\left(s_{1}\right)-f_{i}\left(s_{2}\right)\right| \leq \varrho_{i}\left|s_{1}-s_{2}\right|, \\
\left|g_{i}\left(s_{1}\right)-g_{i}\left(s_{2}\right)\right| \leq \rho_{i}\left|s_{1}-s_{2}\right|, \quad\left|g_{i}(\cdot)\right| \leq G_{i} .
\end{gathered}
$$

\section{Existence of Periodic Solution}

In this section, we will give a sufficient condition which ensures the existence of periodic solution of memristor-based neural network (1).

Theorem 8. Under assumptions $\left(A_{1}\right)$ and $\left(A_{2}\right)$, if $E_{n}-Q$ is an $M$-matrix, where $Q=\left(q_{i j}\right)_{n \times n}$ and

$$
q_{i j}=\frac{1}{\underline{d}_{i}}\left(\varrho_{j} \widetilde{a}_{i j}+\frac{\rho_{j} \tilde{b}_{i j}}{\sqrt{1-\mu_{i j}}}\right), \quad i, j=1,2, \ldots, n,
$$

Proof. Set $C_{\omega}=\left\{x(t) \in C\left(\mathbb{R}, \mathbb{R}^{n}\right): x(t+\omega)=x(t)\right\}$, $\|x(t)\|_{C_{\omega}}=\sum_{i=1}^{n} \max _{t \in[0, \omega]}\left|x_{i}(t)\right|$. Then $C_{\omega}$ is a Banach space with the norm $\|\cdot\|_{C_{\omega}}$. Let $x(t)=\left(x_{1}(t), x_{2}(t), \ldots, x_{n}(t)\right)^{T} \epsilon$ $C_{\omega}, F(t, x)=\left(F_{1}(t, x), F_{2}(t, x), \ldots, F_{n}(t, x)\right)^{T}$, where

$$
\begin{aligned}
F_{i}(t, x)= & -\operatorname{co}\left(d_{i}\left(x_{i}(t)\right)\right) x_{i}(t) \\
& +\sum_{j=1}^{n} \operatorname{co}\left(a_{i j}\left(x_{i}(t)\right)\right) f_{j}\left(x_{j}(t)\right) \\
& +\sum_{j=1}^{n} \operatorname{co}\left(b_{i j}\left(x_{i}(t)\right)\right) g_{j}\left(x_{j}\left(t-\tau_{i j}(t)\right)\right)+I_{i}(t),
\end{aligned}
$$

$i=1,2, \ldots, n$. It is obvious that the set-valued map $F(t, x)$ has nonempty compact convex values. Futhermore, it is USC.

Based on the conditions of Lemma 7, the proof will be divided into three steps.

Step 1. We need to search for appropriate open bounded subset $\Omega$. Assume that $x(t)=\left(x_{1}(t), x_{2}(t), \ldots, x_{n}(t)\right)^{T}$ is an arbitrary $\omega$-periodic solution of differential inclusion $\dot{x}(t) \epsilon$ $\lambda F(t, x)$ for a certain $\lambda \in(0,1)$. Then, one has

$$
\begin{aligned}
\dot{x}_{i}(t) \in \lambda[ & -\operatorname{co}\left(d_{i}\left(x_{i}(t)\right)\right) x_{i}(t) \\
& +\sum_{j=1}^{n} \operatorname{co}\left(a_{i j}\left(x_{i}(t)\right)\right) f_{j}\left(x_{j}(t)\right) \\
& \left.+\sum_{j=1}^{n} \operatorname{co}\left(b_{i j}\left(x_{i}(t)\right)\right) g_{j}\left(x_{j}\left(t-\tau_{i j}(t)\right)\right)+I_{i}(t)\right],
\end{aligned}
$$

or equivalently, there exist measurable functions $d_{i}(t) \epsilon$ $\operatorname{co}\left(d_{i}\left(x_{i}(t)\right)\right), a_{i j}(t) \in \operatorname{co}\left(a_{i j}\left(x_{i}(t)\right)\right)$, and $b_{i j}(t) \in \operatorname{co}\left(b_{i j}\left(x_{i}(t)\right)\right)$, such that

$$
\begin{aligned}
\dot{x}_{i}(t)=\lambda[- & d_{i}(t) x_{i}(t)+\sum_{j=1}^{n} a_{i j}(t) f_{j}\left(x_{j}(t)\right) \\
& \left.+\sum_{j=1}^{n} b_{i j}(t) g_{j}\left(x_{j}\left(t-\tau_{i j}(t)\right)\right)+I_{i}(t)\right],
\end{aligned}
$$

for $i=1,2, \ldots, n$. Multiplying both sides of (14) by $x_{i}(t)$ and integrating over the interval $[0, \omega]$, one has

$$
\begin{aligned}
& \int_{0}^{\omega} d_{i}(t) x_{i}^{2}(t) d t \\
&=\int_{0}^{\omega} x_{i}(t)\left[\sum_{j=1}^{n} a_{i j}(t) f_{j}\left(x_{j}(t)\right)\right. \\
&\left.+\sum_{j=1}^{n} b_{i j}(t) g_{j}\left(x_{j}\left(t-\tau_{i j}(t)\right)\right)+I_{i}(t)\right] d t .
\end{aligned}
$$

then system (1) has at least one $\omega$-periodic solution. 
Noting that

$$
\begin{aligned}
& \int_{0}^{\omega}\left|x_{j}\left(t-\tau_{i j}(t)\right)\right| d t \\
& \quad=\int_{-\tau_{i j}(0)}^{\omega-\tau_{i j}(\omega)} \frac{\left|x_{j}(t)\right|}{1-\dot{\tau}_{i j}\left(\kappa_{i j}^{-1}(t)\right)} d t \\
& \quad=\int_{0}^{\omega} \frac{\left|x_{j}(t)\right|}{1-\dot{\tau}_{i j}\left(\kappa_{i j}^{-1}(t)\right)} d t \leq \frac{1}{1-\mu_{i j}} \int_{0}^{\omega}\left|x_{j}(t)\right| d t
\end{aligned}
$$

where $\kappa_{i j}^{-1}$ is the inverse function of $\kappa_{i j}(t)=t-\tau_{i j}(t), i, j=$ $1,2, \ldots, n$, from (15) and (16), it yields

$$
\begin{aligned}
\underline{d}_{i} \int_{0}^{\omega}\left|x_{i}(t)\right|^{2} d t \\
\leq \sum_{j=1}^{n} \widetilde{a}_{i j} \int_{0}^{\omega}\left|x_{i}(t)\right|\left|f_{j}\left(x_{j}(t)\right)\right| d t \\
\quad+\sum_{j=1}^{n} \widetilde{b}_{i j} \int_{0}^{\omega}\left|x_{i}(t)\right|\left|g_{j}\left(x_{j}\left(t-\tau_{i j}(t)\right)\right)\right| d t \\
\quad+\widetilde{I}_{i} \int_{0}^{\omega}\left|x_{i}(t)\right| d t \\
\leq \sum_{j=1}^{n} \widetilde{a}_{i j} \varrho_{j} \int_{0}^{\omega}\left|x_{i}(t)\right|\left|x_{j}(t)\right| d t \\
\quad+\sum_{j=1}^{n} \widetilde{b}_{i j} \rho_{j} \int_{0}^{\omega}\left|x_{i}(t)\right|\left|x_{j}\left(t-\tau_{i j}(t)\right)\right| d t \\
\quad+\sum_{j=1}^{n}\left(\widetilde{I}_{i} \sqrt{\omega}_{i j} \varrho_{j}+\frac{\tilde{b}_{i j} \rho_{j}}{1-\mu_{i j}}\right)\left(\left.\int_{0}^{\omega}\left|x_{i} \int_{0}^{\omega}\right| x_{i}(t)\right|^{2} d t\right)^{1 / 2}, \\
\quad+\widetilde{I}_{i} \int_{0}^{\omega}\left|x_{i}(t)\right| d t \\
\leq \sum_{j=1}^{n}\left(\tilde{a}_{i j} \varrho_{j}+\frac{\tilde{b}_{i j} \rho_{j}}{1-\mu_{i j}}\right) \int_{0}^{\omega}\left|x_{i}(t)\right|\left|x_{j}(t)\right| d t
\end{aligned}
$$

where $\widetilde{I}_{i}=\sup _{t \in[0, \omega]}\left|I_{i}(t)\right|$. This means

$$
\left(\int_{0}^{\omega}\left|x_{i}(t)\right|^{2} d t\right)^{1 / 2} \leq \sum_{j=1}^{n} q_{i j}\left(\int_{0}^{\omega}\left|x_{j}(t)\right|^{2} d t\right)^{1 / 2}+\frac{\sqrt{\omega} \widetilde{I}_{i}}{\underline{d}_{i}} .
$$

Define $\left\|x_{i}\right\|_{2}^{\omega}=\left(\int_{0}^{\omega}\left|x_{i}(t)\right|^{2} d t\right)^{1 / 2}, x_{i} \in C(\mathbb{R}, \mathbb{R}), i=1,2, \ldots, n$, and $I_{N}=\left(\widetilde{I}_{1} / \underline{d}_{1}, \widetilde{I}_{2} / \underline{d}_{2}, \ldots, \widetilde{I}_{n} / \underline{d}_{n}\right)^{T}$. From (18), we have

$$
\left(E_{n}-Q\right)\left(\left\|x_{1}\right\|_{2}^{\omega},\left\|x_{2}\right\|_{2}^{\omega}, \ldots,\left\|x_{n}\right\|_{2}^{\omega}\right)^{T} \leq \sqrt{\omega} I_{N} .
$$

Since $E_{n}-Q$ is an $M$-matrix, we can choose a vector $\xi=$ $\left(\xi_{1}, \xi_{2}, \ldots, \xi_{n}\right)>(0,0, \ldots, 0)$ such that

$$
\xi^{*}=\left(\xi_{1}^{*}, \xi_{2}^{*}, \ldots, \xi_{n}^{*}\right)=\xi\left(E_{n}-Q\right)>(0,0, \ldots, 0) .
$$

By combining (19) and (20) together, one can derive

$$
\begin{gathered}
\min \left\{\xi_{1}^{*}, \xi_{2}^{*}, \ldots, \xi_{n}^{*}\right\}\left(\left\|x_{1}\right\|_{2}^{\omega}+\left\|x_{2}\right\|_{2}^{\omega}+\cdots+\left\|x_{n}\right\|_{2}^{\omega}\right) \\
\leq \xi_{1}^{*}\left\|x_{1}\right\|_{2}^{\omega}+\xi_{2}^{*}\left\|x_{2}\right\|_{2}^{\omega}+\cdots+\xi_{n}^{*}\left\|x_{n}\right\|_{2}^{\omega} \\
=\xi\left(E_{n}-Q\right)\left(\left\|x_{1}\right\|_{2}^{\omega},\left\|x_{2}\right\|_{2}^{\omega}, \ldots,\left\|x_{n}\right\|_{2}^{\omega}\right)^{T} \\
\leq \xi \sqrt{\omega}\left(\frac{\widetilde{I}_{1}}{\underline{d}_{1}}, \frac{\widetilde{I}_{2}}{\underline{d}_{2}}, \ldots, \frac{\widetilde{I}_{n}}{\underline{d}_{n}}\right)^{T}=\sqrt{\omega} \sum_{i=1}^{n} \xi_{i} \frac{\widetilde{I}_{i}}{\underline{d}_{i}} .
\end{gathered}
$$

Thus, we can easily get that

$$
\left\|x_{i}\right\|_{2}^{\omega}=\left(\int_{0}^{\omega}\left|x_{i}(t)\right|^{2} d t\right)^{1 / 2} \leq \sqrt{\omega} N, \quad i=1,2, \ldots, n,
$$

where $N=\sum_{i=1}^{n}\left(\xi_{i} \widetilde{I}_{i} / \underline{d}_{i}\right) / \min \left\{\xi_{1}^{*}, \xi_{2}^{*}, \ldots, \xi_{n}^{*}\right\}$. Then, there exists $t^{*} \in[0, \omega]$ such that

$$
\left|x_{i}\left(t^{*}\right)\right| \leq N, \quad i=1,2, \ldots, n .
$$

Obviously, for $t \in[0, \omega], x_{i}(t)=x_{i}\left(t^{*}\right)+\int_{t^{*}}^{t} \dot{x}_{i}(s) d s$. It follows from (23) that

$$
\left|x_{i}(t)\right| \leq N+\int_{0}^{\omega}\left|\dot{x}_{i}(t)\right| d t, \quad i=1,2, \ldots, n .
$$

On the other hand, from (14), one easily obtains that

$$
\begin{aligned}
\int_{0}^{\omega}\left|\dot{x}_{i}(t)\right| d t< & \int_{0}^{\omega}\left|d_{i}(t)\right|\left|x_{i}(t)\right| d t \\
& +\sum_{j=1}^{n} \int_{0}^{\omega}\left|a_{i j}(t)\right|\left|f_{j}\left(x_{j}(t)\right)\right| d t \\
& +\sum_{j=1}^{n} \int_{0}^{\omega}\left|b_{i j}(t)\right|\left|g_{j}\left(x_{j}\left(t-\tau_{i j}(t)\right)\right)\right| d t \\
& +\int_{0}^{\omega}\left|I_{i}(t)\right| d t \\
\leq & \bar{d}_{i} \int_{0}^{\omega}\left|x_{i}(t)\right| d t+\sum_{j=1}^{n} \widetilde{a}_{i j} \int_{0}^{\omega}\left|f_{j}\left(x_{j}(t)\right)\right| d t \\
& +\sum_{j=1}^{n} \widetilde{b}_{i j} \int_{0}^{\omega}\left|g_{j}\left(x_{j}\left(t-\tau_{i j(t)}\right)\right)\right| d t+\omega \widetilde{I}_{i}
\end{aligned}
$$




$$
\begin{aligned}
& \leq \bar{d}_{i} \int_{0}^{\omega}\left|x_{i}(t)\right| d t+\sum_{j=1}^{n} \widetilde{a}_{i j} \varrho_{j} \int_{0}^{\omega}\left|x_{j}(t)\right| d t \\
& \quad+\sum_{j=1}^{n} \widetilde{b}_{i j} \rho_{j} \int_{0}^{\omega}\left|x_{j}\left(t-\tau_{i j}(t)\right)\right| d t+\omega \widetilde{I}_{i} \\
& \leq \bar{d}_{i} \sqrt{\omega}\left\|x_{i}\right\|_{2}^{\omega} \\
& \quad+\sum_{j=1}^{n}\left(\varrho_{j} \widetilde{a}_{i j}+\frac{\rho_{j} \widetilde{b}_{i j}}{\sqrt{1-\mu_{i j}}}\right) \sqrt{\omega}\left\|x_{j}\right\|_{2}^{\omega}+\omega \widetilde{I}_{i} \\
& \leq \sqrt{\omega} N\left(\bar{d}_{i}+\underline{d}_{i} \sum_{j=1}^{n} q_{i j}\right)+\omega \widetilde{I}_{i} .
\end{aligned}
$$

Let $M_{i}=\sqrt{\omega} N\left(\bar{d}_{i}+\underline{d}_{i} \sum_{j=1}^{n} q_{i j}\right)+\omega \widetilde{I}_{i}$; combining (24) and (25), we can derive

$$
\left|x_{i}(t)\right|<N+M_{i}=R_{i}, \quad i=1,2, \ldots, n .
$$

Clearly, $R_{i}$ is independent of $\lambda$. In addition, since $E_{n}-Q$ is an $M$-matrix, there exists a vector $\eta=\left(\eta_{1}, \eta_{2}, \ldots, \eta_{n}\right)^{T}>$ $(0,0, \ldots, 0)^{T}$ such that $\left(E_{n}-Q\right) \eta>(0,0, \ldots, 0)^{T}$. Thus, we can choose a sufficiently large constant $\theta$ such that $\eta^{*}=$ $\left(\eta_{1}^{*}, \eta_{2}^{*}, \ldots, \eta_{n}^{*}\right)^{T}=\left(\theta \eta_{1}, \theta \eta_{2}, \ldots, \theta \eta_{n}\right)^{T}=\theta \eta, \eta_{i}^{*}=\theta \eta_{i}>R_{i}$, and

$$
\left(E_{n}-Q\right) \eta^{*}>I_{N}
$$

Taking $\Omega=\left\{x(t) \in C_{\omega} \mid-\eta^{*}<x(t)<\eta^{*}\right\}$, then, $\Omega$ is an open bounded set of $C_{\omega}$ and $x \notin \partial \Omega$ for any $\lambda \in(0,1)$. This proves that condition (1) in Lemma 7 is satisfied.

Step 2. Suppose that there exists a solution $u=\left(u_{1}, u_{2}, \ldots\right.$, $\left.u_{n}\right)^{T} \in \partial \Omega \bigcap \mathbb{R}^{n}$ of the inclusion $0 \in(1 / \omega) \int_{0}^{\omega} F(t, u) d t=$ $\varpi(u)$; then $u$ is a constant vector on $\mathbb{R}^{n}$ such that $\left|u_{i}\right|=\eta_{i}^{*}$ for some $i \in\{1,2, \ldots, n\}$. Therefore, one has

$$
\begin{aligned}
0 \in & (\omega(u))_{i} \\
= & -\frac{u_{i}}{\omega} \int_{0}^{\omega} \operatorname{co}\left(d_{i}\left(x_{i}(t)\right)\right) d t \\
& +\sum_{j=1}^{n} \frac{1}{\omega} \int_{0}^{\omega} \operatorname{co}\left(a_{i j}\left(x_{i}(t)\right)\right) f_{j}\left(u_{j}\right) d t \\
& +\sum_{j=1}^{n} \frac{1}{\omega} \int_{0}^{\omega} \operatorname{co}\left(b_{i j}\left(x_{i}(t)\right)\right) g_{j}\left(u_{j}\right) d t \\
& +\frac{1}{\omega} \int_{0}^{\omega} I_{i}(t) d t,
\end{aligned}
$$

or equivalently, there exist $d_{i}(t) \in \operatorname{co}\left(d_{i}\left(x_{i}(t)\right)\right), a_{i j}(t) \in$ $\operatorname{co}\left(a_{i j}\left(x_{i}(t)\right)\right)$, and $b_{i j}(t) \in \operatorname{co}\left(b_{i j}\left(x_{i}(t)\right)\right)$, such that

$$
\begin{aligned}
0= & -\frac{u_{i}}{\omega} \int_{0}^{\omega} d_{i}(t) d t+\sum_{j=1}^{n} \frac{f_{j}\left(u_{j}\right)}{\omega} \int_{0}^{\omega} a_{i j}(t) d t \\
& +\sum_{j=1}^{n} \frac{g_{j}\left(u_{j}\right)}{\omega} \int_{0}^{\omega} b_{i j}(t) d t+\frac{1}{\omega} \int_{0}^{\omega} I_{i}(t) d t .
\end{aligned}
$$

Then, there exists $t_{*} \in[0, \omega]$ such that

$$
\begin{aligned}
0= & -u_{i} d_{i}\left(t_{*}\right)+\sum_{j=1}^{n} a_{i j}\left(t_{*}\right) f_{j}\left(u_{j}\right) \\
& +\sum_{j=1}^{n} b_{i j}\left(t_{*}\right) g_{j}\left(u_{j}\right)+I_{i}\left(t_{*}\right) .
\end{aligned}
$$

It follows from (30) that

$$
\begin{aligned}
\eta_{i}^{*} & =\left|u_{i}\right| \\
& \leq \frac{1}{\underline{d}_{i}}\left[\sum_{j=1}^{n}\left(\widetilde{a}_{i j} e_{j}+\widetilde{b}_{i j} \rho_{j}\right)\left|u_{j}\right|+\widetilde{I}_{i}\right] \\
& \leq \sum_{j=1}^{n} q_{i j}\left|u_{j}\right|+\frac{\widetilde{I}_{i}}{\underline{d}_{i}} \\
& =\sum_{j=1}^{n} q_{i j} \eta_{j}^{*}+\frac{\widetilde{I}_{i}}{\underline{d}_{i}} .
\end{aligned}
$$

This means $\left(E_{n}-Q\right) \eta^{*} \leq I_{N}$, which contradicts (27).

Step 3. We define a homotopic set-valued map $\phi: \Omega \bigcap \mathbb{R}^{n} \times$ $[0,1] \rightarrow C_{\omega}$ by $\phi(u, h)=-\operatorname{diag}\left(h \bar{d}_{1}, h \bar{d}_{2}, \ldots, h \bar{d}_{n}\right) u+(1-$ h) $\omega(u)$.

If $u=\left(u_{1}, u_{2}, \ldots, u_{n}\right)^{T} \in \partial \Omega \bigcap \mathbb{R}^{n}$, then $u$ is a constant vector on $\mathbb{R}^{n}$ such that $\left|u_{i}\right|=\eta_{i}^{*}$ for some $i \in\{1,2, \ldots, n\}$. It follows that

$$
\begin{aligned}
(\phi(u, h))_{i} & \\
=-h \bar{d}_{i} u_{i} & \\
+(1-h)[ & -\operatorname{co}\left(d_{i}\left(x_{i}(t)\right)\right) u_{i} \\
& +\sum_{j=1}^{n} \frac{f_{j}\left(u_{j}\right)}{\omega} \int_{0}^{\omega} \operatorname{co}\left(a_{i j}\left(x_{i}(t)\right)\right) d t \\
& +\sum_{j=1}^{n} \frac{g_{j}\left(u_{j}\right)}{\omega} \int_{0}^{\omega} \operatorname{co}\left(b_{i j}\left(x_{i}(t)\right)\right) d t \\
& \left.+\frac{1}{\omega} \int_{0}^{\omega} I_{i}(t) d t\right] .
\end{aligned}
$$


In fact, we have $0 \notin(\phi(u, h))_{i}, i \in\{1,2, \ldots, n\}$. If $0 \in$ $(\phi(u, h))_{i}$, that is,

$$
\begin{aligned}
& 0 \in-h \bar{d}_{i} u_{i} \\
&+(1-h)\left[-\operatorname{co}\left(d_{i}\left(x_{i}(t)\right)\right) u_{i}\right. \\
&+\sum_{j=1}^{n} \frac{f_{j}\left(u_{j}\right)}{\omega} \int_{0}^{\omega} \operatorname{co}\left(a_{i j}\left(x_{i}(t)\right)\right) d t \\
&+\sum_{j=1}^{n} \frac{g_{j}\left(u_{j}\right)}{\omega} \int_{0}^{\omega} \operatorname{co}\left(b_{i j}\left(x_{i}(t)\right)\right) d t \\
&\left.+\frac{1}{\omega} \int_{0}^{\omega} I_{i}(t) d t\right],
\end{aligned}
$$

or

$$
\begin{aligned}
& 0=-h \bar{d}_{i} u_{i} \\
&+(1-h)\left[-d_{i}(t) u_{i}+\sum_{j=1}^{n} \frac{f_{j}\left(u_{j}\right)}{\omega} \int_{0}^{\omega} a_{i j}(t) d t\right. \\
& \\
&\left.+\sum_{j=1}^{n} \frac{g_{j}\left(u_{j}\right)}{\omega} \int_{0}^{\omega} b_{i j}(t) d t+\frac{1}{\omega} \int_{0}^{\omega} I_{i}(t) d t\right] .
\end{aligned}
$$

There exists $t_{0} \in[0, \omega]$, such that

$$
\begin{aligned}
0=- & -h \bar{d}_{i} u_{i} \\
& +(1-h)\left[-d_{i}\left(t_{0}\right) u_{i}+\sum_{j=1}^{n} a_{i j}\left(t_{0}\right) f_{j}\left(u_{j}\right)\right. \\
& \left.+\sum_{j=1}^{n} b_{i j}\left(t_{0}\right) g_{j}\left(u_{j}\right)+I_{i}\left(t_{0}\right)\right],
\end{aligned}
$$

that is,

$$
\begin{aligned}
& h\left(\bar{d}_{i}-d_{i}\left(t_{0}\right)\right) u_{i}+d_{i}\left(t_{0}\right) u_{i} \\
& =(1-h)\left[\sum_{j=1}^{n}\left(a_{i j}\left(t_{0}\right) f_{j}\left(u_{j}\right)+b_{i j}\left(t_{0}\right) g_{j}\left(u_{j}\right)\right)+I_{i}\left(t_{0}\right)\right] .
\end{aligned}
$$

Therefore, we have

$$
\begin{aligned}
\eta_{i}^{*} & =\left|u_{i}\right| \\
& \leq \frac{1-h}{h\left(\bar{d}_{i}-d_{i}\left(t_{0}\right)\right)+d_{i}\left(t_{0}\right)}
\end{aligned}
$$

$$
\begin{aligned}
& \quad \times\left[\sum_{j=1}^{n}\left(\left|a_{i j}\left(t_{0}\right)\right|\left|f_{j}\left(u_{j}\right)\right|+\left|b_{i j}\left(t_{0}\right)\right|\left|g_{j}\left(u_{j}\right)\right|\right)\right. \\
& \left.\quad+\left|I_{i}\left(t_{0}\right)\right|\right] \\
& \leq \frac{1}{\underline{d}_{i}}\left[\sum_{j=1}^{n}\left(\widetilde{a}_{i j} e_{j}+\widetilde{b}_{i j} \rho_{j}\right)\left|u_{j}\right|+\widetilde{I}_{i}\right] \\
& \leq \sum_{j=1}^{n} q_{i j}\left|u_{j}\right|+\frac{\widetilde{I}_{i}}{\underline{d}_{i}} \\
& =\sum_{j=1}^{n} q_{i j} \eta_{i}^{*}+\frac{\widetilde{I}_{i}}{\underline{d}_{i}}
\end{aligned}
$$

This means that $\left(E_{n}-Q\right) \eta^{*} \leq I_{N}$, which contradicts (27). Thus, $0 \notin(\phi(u, h))_{i}, i=1,2, \ldots, n$. It follows that $(0,0, \ldots, 0)^{T} \notin \phi(u, h)$, for any $u=\left(u_{1}, u_{2}, \ldots, u_{n}\right)^{T} \epsilon$ $\partial \Omega \bigcap \mathbb{R}^{n}, h \in[0,1]$. Therefore, by the homotopy invariance and the solution properties of the topological degree, one has

$$
\begin{aligned}
& \operatorname{deg}\left\{\emptyset, \Omega \bigcap \mathbb{R}^{n}, 0\right\}=\operatorname{deg}\left\{\phi(u, 0), \Omega \bigcap \mathbb{R}^{n}, 0\right\} \\
& =\operatorname{deg}\left\{\phi(u, 1), \Omega \bigcap \mathbb{R}^{n}, 0\right\} \\
& =\operatorname{deg}\left\{\left(-\bar{d}_{1} u_{1},-\bar{d}_{2} u_{2}, \ldots,-\bar{d}_{n} u_{n}\right)^{T}, \Omega \bigcap \mathbb{R}^{n},(0,0, \ldots, 0)^{T}\right\} \\
& =\operatorname{sign}\left|\begin{array}{ccc}
-\bar{d}_{1} & \cdots & 0 \\
\vdots & \ddots & \vdots \\
0 & \ldots & -\bar{d}_{n}
\end{array}\right|=(-1)^{n} \neq 0,
\end{aligned}
$$

where $\operatorname{deg}(\cdot, \cdot, \cdot)$ denotes the topological degree for USC setvalued maps with compact convex values.

Up to now, we have proved that $\Omega$ satisfies all the conditions in Lemma 7, then system (1) has at least one $\omega$ periodic solution. This completes the proof.

Notice that a constant function can be regarded as a special periodic function with arbitrary period or zero amplitude. Hence, we can obtain the following result.

Corollary 9. Suppose assumption $\left(A_{2}\right)$ holds and $I_{i}(t)=I_{i}$, $\tau_{i j}(t)=\tau_{i j}$, if $E_{n}-Q$ is an M-matrix, where $Q=\left(q_{i j}\right)_{n \times n}$ and

$$
q_{i j}=\frac{1}{\underline{d}_{i}}\left(\varrho_{j} \widetilde{a}_{i j}+\rho_{j} \widetilde{b}_{i j}\right), \quad i, j=1,2, \ldots, n,
$$

then system (1) exists at least one equilibrium point.

Remark 10. By employing the method based on the $M$ matrix theory, our results can be easily verified and are much different from these in the literature [20,21]. It is also worth mentioning that the $M$-matrix theory is one of the effective 
and important methods to deal with the existence of periodic solution and equilibrium point for large-scale dynamical neuron systems.

\section{Complete Periodic Synchronization}

In this paper, we consider model (1) as the master system, and a slave system for (1) can be described by the following equation:

$$
\begin{array}{r}
\dot{y}_{i}(t)=-d_{i}\left(y_{i}(t)\right) x_{i}(t)+\sum_{j=1}^{n} a_{i j}\left(y_{i}(t)\right) f_{j}\left(y_{j}(t)\right) \\
+\sum_{j=1}^{n} b_{i j}\left(y_{i}(t)\right) g_{j}\left(y_{j}\left(t-\tau_{i j}(t)\right)\right)+I_{i}(t)+u_{i}(t), \\
t \geq 0, i=1,2, \ldots, n,
\end{array}
$$

where $u_{i}(t)$ is the controller to be designed, and

$$
\begin{aligned}
& d_{i}\left(y_{i}(t)\right)= \begin{cases}\dot{d}_{i}, & \left|y_{i}(t)\right| \leq T_{i}, \\
\grave{d}_{i}, & \left|y_{i}(t)\right|>T_{i},\end{cases} \\
& a_{i j}\left(y_{i}(t)\right)= \begin{cases}\dot{a}_{i j}, & \left|y_{i}(t)\right| \leq T_{i}, \\
\grave{a}_{i j}, & \left|y_{i}(t)\right|>T_{i},\end{cases} \\
& b_{i j}\left(y_{i}(t)\right)= \begin{cases}\hat{b}_{i j}, & \left|y_{i}(t)\right| \leq T_{i}, \\
\dot{b}_{i j}, & \left|y_{i}(t)\right|>T_{i} .\end{cases}
\end{aligned}
$$

Let $e_{i}(t)=y_{i}(t)-x_{i}(t), i=1,2, \ldots, n$; one can obtain the following result.

Theorem 11. Suppose that all the conditions of Theorem 8 are satisfied; then the salve system (40) can globally synchronize with the master system (1) under the following adaptive controller:

$$
\begin{gathered}
u_{i}(t)=-\alpha_{i}(t) e_{i}(t)-\delta_{i} \beta_{i}(t) \operatorname{sign}\left(e_{i}(t)\right), \\
\dot{\alpha}_{i}(t)=-\varepsilon_{i} e_{i}^{2}(t), \quad i=1,2, \ldots, n, \\
\dot{\beta}_{i}(t)=-\eta_{i}\left|e_{i}(t)\right|, \quad i=1,2, \ldots, n,
\end{gathered}
$$

where $\alpha_{i}, \eta_{i}$ are arbitrary positive constants and $\delta_{i}>1$.

Proof. Consider the following Lyapunov functional:

$$
\begin{aligned}
V(t)= & \frac{1}{2} \sum_{i=1}^{n} e_{i}^{2}(t)+\sum_{i, j=1}^{n} \frac{1}{2\left(1-\mu_{i j}\right)} \int_{t-\tau_{i j}(t)}^{t} e_{i}^{2}(s) d s \\
& +\sum_{i=1}^{n} \frac{1}{2 \varepsilon_{i}}\left(\alpha_{i}(t)-K_{i}\right)^{2}+\sum_{i=1}^{n} \frac{1}{2 \eta_{i}}\left(M_{i}-\beta_{i}(t)\right)^{2}
\end{aligned}
$$

where

$$
\begin{gathered}
K_{i} \geq \max \left\{-\dot{d}_{i}+\sum_{j=1}^{n} \frac{\varrho_{j}^{2} \dot{a}_{i j}^{2}+\rho_{j}^{2} \dot{b}_{i j}^{2}+1}{2}+\sum_{j=1}^{n} \frac{1}{2\left(1-\mu_{j i}\right)},-\grave{d}_{i}\right. \\
\left.+\sum_{j=1}^{n} \frac{\varrho_{j}^{2} \grave{a}_{i j}^{2}+\rho_{j}^{2} \grave{b}_{i j}^{2}+1}{2}+\sum_{j=1}^{n} \frac{1}{2\left(1-\mu_{j i}\right)}\right\}, \\
M_{i} \geq\left|\grave{d}_{i}-\grave{d}_{i}\right| T_{i}+\sum_{j=1}^{n} \varrho_{j}\left|\dot{a}_{i j}-\grave{a}_{i j}\right| T_{i}+\sum_{j=1}^{n}\left|\dot{b}_{i j}-\grave{b}_{i j}\right| G_{j} .
\end{gathered}
$$

The master system (1) and the slave system (40) are statedependent switching systems; then, the four cases may appear in the following at time $t$.

Case 1. If $\left|x_{i}(t)\right| \leq T_{i},\left|y_{i}(t)\right| \leq T_{i}$ at time $t$, then the master system (1) and the slave system (40) reduce to the following systems, respectively,

$$
\begin{aligned}
\dot{x}_{i}(t)= & -\dot{d}_{i} x_{i}(t)+\sum_{j=1}^{n} \dot{a}_{i j} f_{j}\left(x_{j}(t)\right) \\
& +\sum_{i=j}^{n} \dot{b}_{i j} g_{j}\left(x_{j}\left(t-\tau_{i j}(t)\right)\right)+I_{i}(t), \\
\dot{y}_{i}(t)= & -\dot{d}_{i} y_{i}(t)+\sum_{j=1}^{n} \dot{a}_{i j} f_{j}\left(y_{j}(t)\right) \\
& +\sum_{j=1}^{n} \dot{b}_{i j} g_{j}\left(y_{j}\left(\mathrm{t}-\tau_{i j}(t)\right)\right)+I_{i}(t)+u_{i}(t) .
\end{aligned}
$$

Correspondingly, the error system can be written as

$$
\begin{aligned}
\dot{e}_{i}(t)= & -\dot{d}_{i} e_{i}(t)+\sum_{j=1}^{n} \dot{a}_{i j} f_{j}\left(e_{j}(t)\right) \\
& +\sum_{j=1}^{n} \dot{b}_{i j} g_{j}\left(e_{j}\left(t-\tau_{i j}(t)\right)\right)+u_{i}(t),
\end{aligned}
$$

where $f_{j}\left(e_{j}(t)\right)=f_{j}\left(y_{j}(t)\right)-f_{j}\left(x_{j}(t)\right), g_{j}\left(e_{j}\left(t-\tau_{i j}(t)\right)\right)=$ $g_{j}\left(y_{j}\left(t-\tau_{i j}(t)\right)\right)-g_{j}\left(x_{j}\left(t-\tau_{i j}(t)\right)\right)$. Under assumption $\left(\mathrm{A}_{2}\right)$, evaluating the upper right derivation $D^{+} V(t)$ of $V(t)$ along the trajectory of (47) gives

$D^{+} V(t)$

$$
\begin{aligned}
& \leq \sum_{i=1}^{n}\left\{e _ { i } ( t ) \left[-\dot{d}_{i} e_{i}(t)+\sum_{j=1}^{n} \dot{a}_{i j} f_{j}\left(e_{j}(t)\right)\right.\right. \\
& +\sum_{j=1}^{n} \hat{b}_{i j} g_{j}\left(e_{j}\left(t-\tau_{i j}(t)\right)-\alpha_{i}(t) e_{i}(t)\right. \\
& \left.-\delta_{i} \beta_{i}(t) \operatorname{sign}\left(e_{i}(t)\right)\right] \\
& +\sum_{j=1}^{n}\left(\frac{1}{2\left(1-\mu_{i j}\right)} e_{j}^{2}(t)-\frac{1}{2} e_{j}^{2}\left(t-\tau_{i j}(t)\right)\right)
\end{aligned}
$$




$$
\begin{aligned}
& \left.+\left(\alpha_{i}(t)-K_{i}\right) e_{i}^{2}(t)-\left(M_{i}-\beta_{i}(t)\left|e_{i}(t)\right|\right)\right\} \\
& \leq \sum_{i=1}^{n}\left\{\left[-\hat{d}_{i} e_{i}^{2}(t)+\sum_{j=1}^{n} e_{j}\left|\dot{a}_{i j}\right|\left|e_{i}(t)\right|\left|e_{j}(t)\right|\right.\right. \\
& \left.+\sum_{j=1}^{n} \rho_{j}\left|\hat{b}_{i j}\right|\left|e_{i}(t)\right|\left|e_{j}\left(t-\tau_{i j}(t)\right)\right|-\delta_{i} \beta_{i}(t)\left|e_{i}(t)\right|\right] \\
& +\sum_{j=1}^{n}\left(\frac{1}{2\left(1-\mu_{i j}\right)} e_{j}^{2}(t)-\frac{1}{2} e_{j}^{2}\left(t-\tau_{i j}(t)\right)\right) \\
& \left.-K_{i} e_{i}^{2}(t)-M_{i}\left|e_{i}(t)\right|+\beta_{i}(t)\left|e_{i}(t)\right|\right\} \\
& \leq \sum_{i=1}^{n}\left\{\left[-\left(K_{i}+\dot{d}_{i}\right) e_{i}^{2}(t)+\sum_{j=1}^{n}\left(\frac{1}{2} \varrho_{j}^{2} \dot{a}_{i j}^{2} e_{i}(t)^{2}+\frac{1}{2} e_{j}(t)^{2}\right)\right.\right. \\
& +\sum_{j=1}^{n}\left(\frac{1}{2} \rho_{j}^{2} \dot{b}_{i j}^{2} e_{i}(t)^{2}+\frac{1}{2} e_{j}^{2}\left(t-\tau_{i j}(t)\right)\right) \\
& \left.-\left(\delta_{i}-1\right) \beta_{i}(t)\left|e_{i}(t)\right|\right] \\
& +\sum_{j=1}^{n}\left(\frac{1}{2\left(1-\mu_{i j}\right)} e_{j}^{2}(t)-\frac{1}{2} e_{j}^{2}\left(t-\tau_{i j}(t)\right)\right) \\
& \left.-M_{i}\left|e_{i}(t)\right|\right\} \\
& =\sum_{i=1}^{n}\left\{\left[-K_{i}-\dot{d}_{i}+\sum_{j=1}^{n} \frac{e_{j}^{2} \dot{a}_{i j}^{2}+\rho_{j}^{2} \dot{b}_{i j}^{2}+1}{2}\right.\right. \\
& \left.+\sum_{j=1}^{n} \frac{1}{2\left(1-\mu_{j i}\right)}\right] e_{i}^{2}(t) \\
& \left.-\left(\delta_{i}-1\right) \beta_{i}(t)\left|e_{i}(t)\right|-M_{i}\left|e_{i}(t)\right|\right\} \text {. }
\end{aligned}
$$

Considering the definition of $K_{i}$, and $\delta_{i}>1, M_{i}>0$, one has

$$
D^{+} V(t) \leq 0 \text {. }
$$

Case 2. If $\left|x_{i}(t)\right|>T_{i},\left|y_{i}(t)\right|>T_{i}$ at time $t$, then the master system (1) and the slave system (40) reduce to the following systems, respectively,

$$
\begin{aligned}
\dot{x}_{i}(t)= & -\grave{d}_{i} x_{i}(t)+\sum_{j=1}^{n} \grave{a}_{i j} f_{j}\left(x_{j}(t)\right) \\
& +\sum_{i=j}^{n} \grave{b}_{i j} g_{j}\left(x_{j}\left(t-\tau_{i j}(t)\right)\right)+I_{i}(t),
\end{aligned}
$$

$$
\begin{aligned}
\dot{y}_{i}(t)= & -\grave{d}_{i} y_{i}(t)+\sum_{j=1}^{n} \grave{a}_{i j} f_{j}\left(y_{j}(t)\right) \\
& +\sum_{j=1}^{n} \grave{b}_{i j} g_{j}\left(y_{j}\left(t-\tau_{i j}(t)\right)\right)+I_{i}(t)+u_{i}(t) .
\end{aligned}
$$

Correspondingly, the error system can be rewritten as

$$
\begin{aligned}
\dot{e}_{i}(t)= & -\grave{d}_{i} e_{i}(t)+\sum_{j=1}^{n} \grave{a}_{i j} f_{j}\left(e_{j}(t)\right) \\
& +\sum_{j=1}^{n} \grave{b}_{i j} g_{j}\left(e_{j}\left(t-\tau_{i j}(t)\right)\right)+u_{i}(t) .
\end{aligned}
$$

Arguing as in the proof of Case 1, we can obtain

$D^{+} V(t)$

$$
\begin{aligned}
\leq \sum_{i=1}^{n}\{[ & -K_{i}-\grave{d}_{i}+\sum_{j=1}^{n} \frac{e_{j}^{2} \grave{a}_{i j}^{2}+\rho_{j}^{2} \grave{b}_{i j}^{2}+1}{2} \\
& \left.+\sum_{j=1}^{n} \frac{1}{2\left(1-\mu_{j i}\right)}\right] e_{i}^{2}(t) \\
& \left.-\left(\delta_{i}-1\right) \beta_{i}(t)\left|e_{i}(t)\right|-M_{i}\left|e_{i}(t)\right|\right\} \leq 0 .
\end{aligned}
$$

Case 3. If $\left|x_{i}(t)\right|>T_{i},\left|y_{i}(t)\right| \leq T_{i}$ at time $t$, then the master system (1) and the slave system (40) reduce to (50) and (46). Correspondingly, the error system can be rewritten as

$$
\begin{aligned}
\dot{e}_{i}(t)= & -\grave{d}_{i} e_{i}(t)+\sum_{j=1}^{n} \grave{a}_{i j} f_{j}\left(e_{j}(t)\right)+\sum_{j=1}^{n} \grave{b}_{i j} g_{j}\left(e_{j}\left(t-\tau_{i j}(t)\right)\right) \\
& +\left(\grave{d}_{i}-\dot{d}_{i}\right) y_{i}(t)+\sum_{j=1}^{n}\left(\grave{a}_{i j}-\grave{a}_{i j}\right) f_{j}\left(y_{j}(t)\right) \\
& +\sum_{j=1}^{n}\left(\grave{b}_{i j}-\grave{b}_{i j}\right) g_{j}\left(y_{j}\left(t-\tau_{i j}(t)\right)\right)+u_{i}(t) .
\end{aligned}
$$

Similarly, evaluating the upper right derivation $D^{+} V(t)$ of $V(t)$ along the trajectory of (54), we have

$$
\begin{aligned}
& D^{+} V(t) \\
& \leq \sum_{i=1}^{n}\left\{\left[-K_{i}-\grave{d}_{i}+\sum_{j=1}^{n} \frac{e_{j}^{2} \grave{a}_{i j}^{2}+\rho_{j}^{2} \grave{b}_{i j}^{2}+1}{2}\right.\right. \\
& \left.+\sum_{j=1}^{n} \frac{1}{2\left(1-\mu_{j i}\right)}\right] e_{i}^{2}(t) \\
& +\left|e_{i}(t)\right|\left[\left|\grave{d}_{i}-\hat{d}_{i}\right|\left|y_{i}(t)\right|+\sum_{j=1}^{n}\left|\hat{a}_{i j}-\grave{a}_{i j}\right|\left|f_{j}\left(y_{j}(t)\right)\right|\right.
\end{aligned}
$$




$$
\begin{array}{r}
\left.+\sum_{j=1}^{n}\left|\hat{b}_{i j}-\grave{b}_{i j}\right|\left|g_{j}\left(y_{j}\left(t-\tau_{i j}(t)\right)\right)\right|\right] \\
\left.-\left(\delta_{i}-1\right) \beta_{i}(t)\left|e_{i}(t)\right|-M_{i}\left|e_{i}(t)\right|\right\} .
\end{array}
$$

Note that $\left|y_{i}(t)\right| \leq T_{i}$; by using assumption $\left(\mathrm{A}_{2}\right)$, one has

$$
\begin{aligned}
D^{+} V(t) \leq \sum_{i=1}^{n}\{[ & -K_{i}-\grave{d}_{i}+\sum_{j=1}^{n} \frac{e_{j}^{2} \grave{a}_{i j}^{2}+\rho_{j}^{2} \grave{b}_{i j}^{2}+1}{2} \\
& \left.+\sum_{j=1}^{n} \frac{1}{2\left(1-\mu_{j i}\right)}\right] e_{i}^{2}(t) \\
& +\left|e_{i}(t)\right|\left[\left|\grave{d}_{i}-\grave{d}_{i}\right| T_{i}+\sum_{j=1}^{n} \varrho_{j}\left|\grave{a}_{i j}-\grave{a}_{i j}\right| T_{i}\right. \\
& \left.+\sum_{j=1}^{n}\left|\hat{b}_{i j}-\grave{b}_{i j}\right| G_{j}\right] \\
& \left.-\left(\delta_{i}-1\right) \beta_{i}(t)\left|e_{i}(t)\right|-M_{i}\left|e_{i}(t)\right|\right\} .
\end{aligned}
$$

According to the definition of $K_{i}, M_{i}$, and $\delta_{i}>1$, one has

$$
D^{+} V(t) \leq 0 .
$$

Case 4. If $\left|x_{i}(t)\right| \leq T_{i},\left|y_{i}(t)\right|>T_{i}$ at time $t$, then the master system (1) and the slave system (40) reduce to (45) and (51). Correspondingly, the error system can be rewritten as

$$
\begin{aligned}
\dot{e}_{i}(t)= & -\grave{d}_{i} e_{i}(t)+\sum_{j=1}^{n} \grave{a}_{i j} f_{j}\left(e_{j}(t)\right)+\sum_{j=1}^{n} \grave{b}_{i j} g_{j}\left(e_{j}\left(t-\tau_{i j}(t)\right)\right) \\
& +\left(\grave{d}_{i}-\grave{d}_{i}\right) x_{i}(t)+\sum_{j=1}^{n}\left(\grave{a}_{i j}-\grave{a}_{i j}\right) f_{j}\left(x_{j}(t)\right) \\
& +\sum_{j=1}^{n}\left(\grave{b}_{i j}-\hat{b}_{i j}\right) g_{j}\left(x_{j}\left(t-\tau_{i j}(t)\right)\right)+u_{i}(t) .
\end{aligned}
$$

By using $\left|x_{i}(t)\right| \leq T_{i}$, we can also have

$$
\begin{gathered}
D^{+} V(t) \leq \sum_{i=1}^{n}\left\{\left[-K_{i}-\grave{d}_{i}+\sum_{j=1}^{n} \frac{\varrho_{j}^{2} \grave{a}_{i j}^{2}+\rho_{j}^{2} \grave{b}_{i j}^{2}+1}{2}\right.\right. \\
\left.+\sum_{j=1}^{n} \frac{1}{2\left(1-\mu_{j i}\right)}\right] e_{i}^{2}(t) \\
+\left|e_{i}(t)\right|\left[\left|\grave{d}_{i}-\dot{d}_{i}\right| T_{i}\right.
\end{gathered}
$$

$$
\begin{gathered}
+\sum_{j=1}^{n} \varrho_{j}\left|\dot{a}_{i j}-\grave{a}_{i j}\right| T_{i} \\
\left.+\sum_{j=1}^{n}\left|\dot{b}_{i j}-\grave{b}_{i j}\right| G_{j}\right] \\
\left.-\left(\delta_{i}-1\right) \beta_{i}(t)\left|e_{i}(t)\right|-M_{i}\left|e_{i}(t)\right|\right\} \leq 0 .
\end{gathered}
$$

The above proving procedures clearly imply that one always has $D^{+} V(t) \leq 0$ at time $t$. Therefore, the salve system (40) globally synchronizes with the master system (1) under the adaptive controller (42). This completes the proof.

Remark 12. In the literature, some results on stability analysis of periodic solution (or equilibrium point) or synchronization (or antisynchronization) control of memristor-based neural network were obtained [11-13, 16, 17, 20, 21]. A typical assumption is that

$$
\left[\underline{d}_{i}, \bar{d}_{i}\right] x-\left[\underline{d}_{i}, \bar{d}_{i}\right] y \subseteq\left[\underline{d}_{i}, \bar{d}_{i}\right](x-y), \ldots
$$

However, We can prove that this assumption holds only when $x$ and $y$ have different sign, or $x=0$, or $y=0$. Without this assumption, we divide the error system into four cases in this paper. Under the adaptive controller (42), globally periodic synchronization criterion between system (1) and (40) is derived. The synchronization criterion of this paper which does not solve any inequality or linear matrix inequality is easily verified.

Remark 13. As far as we know, there is no work on the periodic synchronization of memristor-based neural network via adaptive control. Thus, our outcomes are brand new and original compared to the existing results ([11-14]). In addition, the obtained results in this paper are also applicable to the common systems without memristor or the memductance of the memristor equals a constant since they are special cases of memristor-based neural networks.

\section{Numerical Example}

In this section, one example is offered to illustrate the effectiveness of the results obtained in this paper.

Example 1. Consider the second-order memristor-based neural network (1) with the following system parameters:

$$
d_{1}\left(x_{1}(t)\right)= \begin{cases}6.5, & \left|x_{1}(t)\right| \leq \frac{1}{4}, \\ 6, & \left|x_{1}(t)\right|>\frac{1}{4},\end{cases}
$$




$$
\begin{aligned}
& d_{2}\left(x_{2}(t)\right)= \begin{cases}6, & \left|x_{2}(t)\right| \leq \frac{1}{4}, \\
6.5, & \left|x_{2}(t)\right|>\frac{1}{4}\end{cases} \\
& a_{11}\left(x_{1}(t)\right)= \begin{cases}-2, & \left|x_{1}(t)\right| \leq \frac{1}{4} \\
2, & \left|x_{1}(t)\right|>\frac{1}{4}\end{cases} \\
& a_{12}\left(x_{1}(t)\right)= \begin{cases}-1, & \left|x_{1}(t)\right| \leq \frac{1}{4} \\
1, & \left|x_{1}(t)\right|>\frac{1}{4}\end{cases} \\
& a_{21}\left(x_{2}(t)\right)= \begin{cases}1, & \left|x_{2}(t)\right| \leq \frac{1}{4} \\
-1, & \left|x_{2}(t)\right|>\frac{1}{4}\end{cases} \\
& b_{22}\left(x_{2}(t)\right)= \begin{cases}-2, & \left|x_{2}(t)\right| \leq \frac{1}{4}, \\
2, & \left|x_{2}(t)\right|>\frac{1}{4},\end{cases} \\
& b_{21}\left(x_{2}(t)\right)= \begin{cases}1 & \left|x_{2}(t)\right| \leq \frac{1}{4}, \\
-1, & \left|x_{2}(t)\right|>\frac{1}{4},\end{cases} \\
& b_{12}\left(x_{1}(t)\right)= \begin{cases}-1, & \left|x_{1}(t)\right| \leq \frac{1}{4}, \\
1, & \left|x_{1}(t)\right|>\frac{1}{4},\end{cases}
\end{aligned}
$$

and the activation functions are taken as follows:

$$
f_{1}(s)=f_{2}(s)=s, \quad g_{1}(s)=g_{2}(s)=\frac{1}{2} \tanh s .
$$

It can be verified that $\underline{d}_{1}=\underline{d}_{2}=6, \tilde{a}_{11}=\tilde{a}_{22}=2, \tilde{a}_{12}=\tilde{a}_{21}=$ $1, \widetilde{b}_{i j}=1, i, j=1,2, \varrho_{1}=\varrho_{2}=1$, and $\rho_{1}=\rho_{2}=1 / 2$.

We take $\tau_{i j}(t)=3 / 4-(1 / 4) \sin 3 t, i, j=1,2 \mathrm{~A}$ straightforward calculation gives $\tau=1$ and $\mu_{i j}=3 / 4$. Then, we get $E_{2}-Q=\left(\begin{array}{cc}1 / 2 & -1 / 3 \\ -1 / 3 & 1 / 2\end{array}\right)$. Obviously, $E_{2}-Q$ is an $M$-matrix. Thus, the conditions required in Theorem 8 are satisfied. When $I(t)$ is a periodic function, in the view of Theorem 8 , this neural network has at least one periodic solution.

For numerical simulations, we choose the external input $\left(I_{1}(t), I_{2}(t)\right)^{T}=(0.65 \sin 3 t-2,0.65 \cos 3 t+2)^{T}$. The periodic

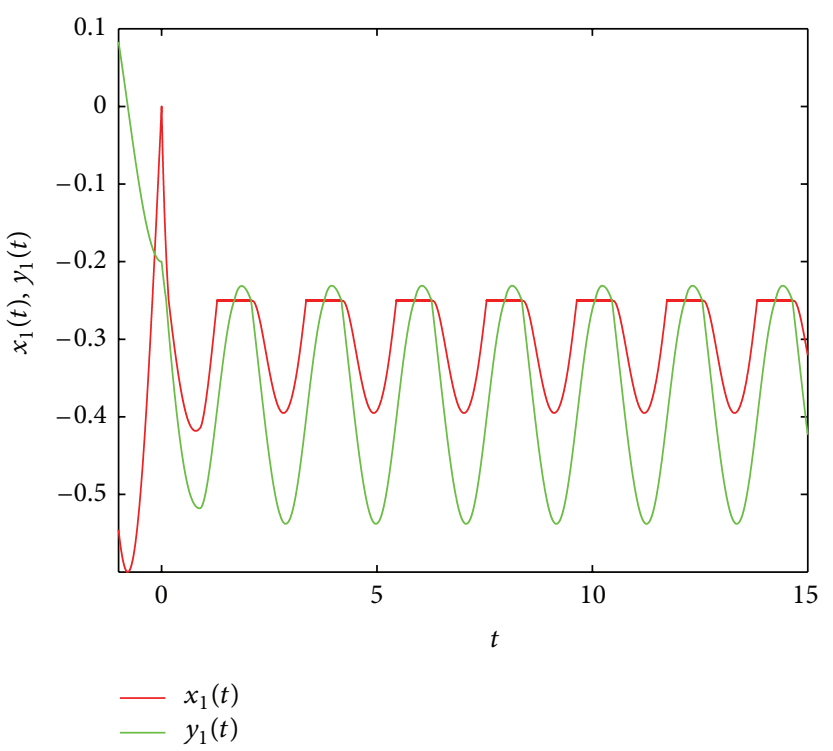

FIGURE 1: Time-domain behavior of the state variables $x_{1}(t)$ and $y_{1}(t)$ with $u_{i}(t)=0$.

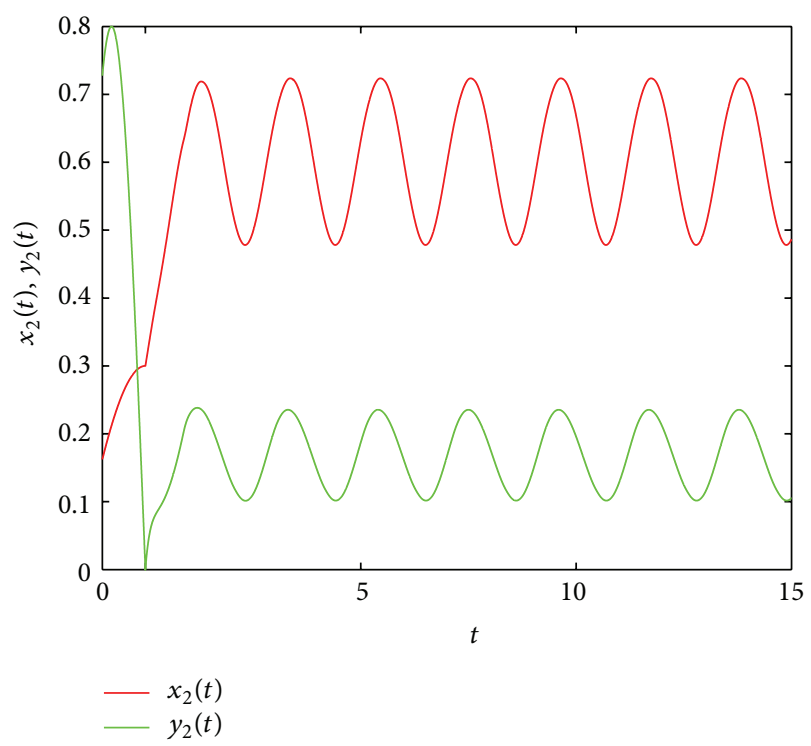

FIgURE 2: Time-domain behavior of the state variables $x_{2}(t)$ and $y_{2}(t)$ with $u_{i}(t)=0$.

dynamic behavior of the master system (1) and the slave system (40) with $u_{i}(t)=0$ is given in Figures 1, 2, and 3, with the initial states chosen as $x(t)=(0.6 \sin 2 t, 0.3 \cos t)^{T}$ and $y(t)=(-0.2 \cos 2 t,-0.8 \sin 2 t)^{T}$ for $t \in[-1,0]$.

In order to demonstrate the adaptive controller (42) can realize complete periodic synchronization of memristorbased neural networks, some initial parameters are taken as $\alpha_{1}(t)=\alpha_{2}(t)=0.2, \beta_{1}(t)=\beta_{2}(t)=0.1$ for $t \in[-1,0]$, $\delta_{i}=10, \varepsilon_{i}=\eta_{i}=0.1, i=1,2$. We get the simulation results shown in Figures 4-6. Figure 4 describes the time responses of synchronization errors $e_{i}(t)=y_{i}(t)-x_{i}(t), i=1,2$, which turn to zero quickly as time goes. Figure 5 shows the time response of $\alpha(t)=\left(\alpha_{1}(t), \alpha_{2}(t)\right)^{T}$. Figure 6 depicts the time 


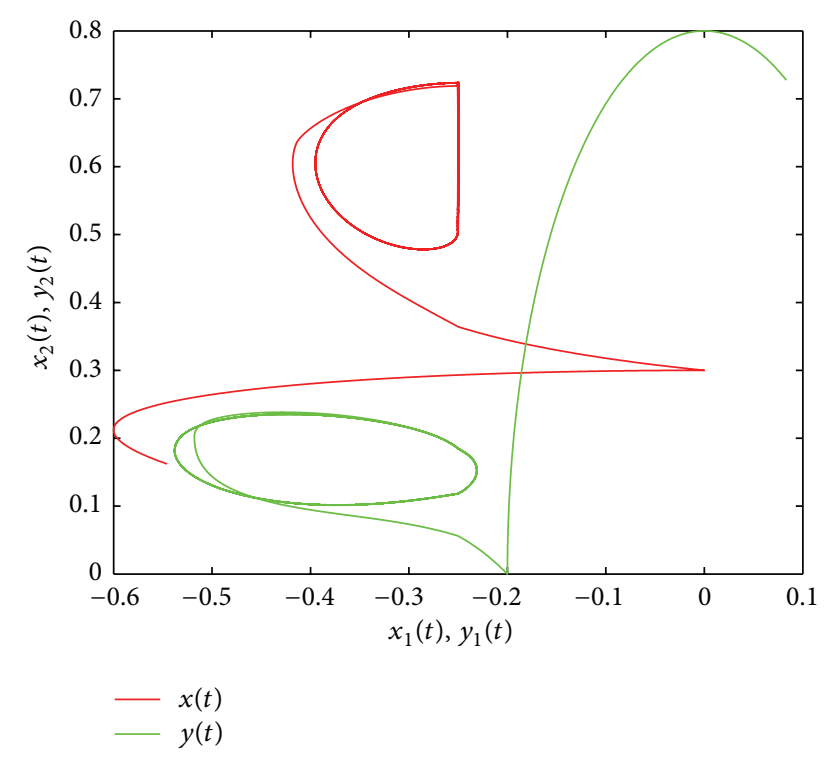

FIGURE 3: Phase plane behavior of the master system (1) and the salve system (40) with $u_{i}=0$.

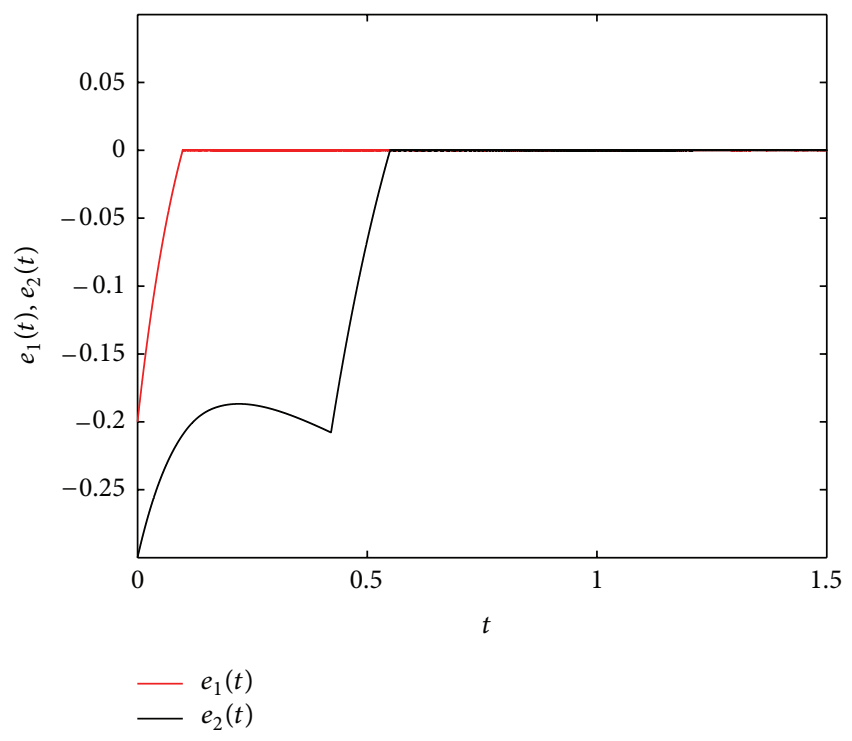

Figure 4: The synchronization errors $e_{1}(t)$ and $e_{2}(t)$ under the adaptive controller (42).

response of $\beta(t)=\left(\beta_{1}(t), \beta_{2}(t)\right)^{T}$. From Figures 5 and 6 one can see that the control parameters $\alpha_{i}(t)$ and $\beta_{i}(t), i=1,2$, turn out to be some constants eventually.

\section{Conclusion}

In this paper, complete periodic synchronization of a class of memristor-based neural networks has been investigated. The master system synchronizes with the slave system by using adaptive control. The obtained results are novel since there are few works about complete periodic synchronization issue of memristor-based neural networks via adaptive control. In addition, the easily testable condition which ensures the

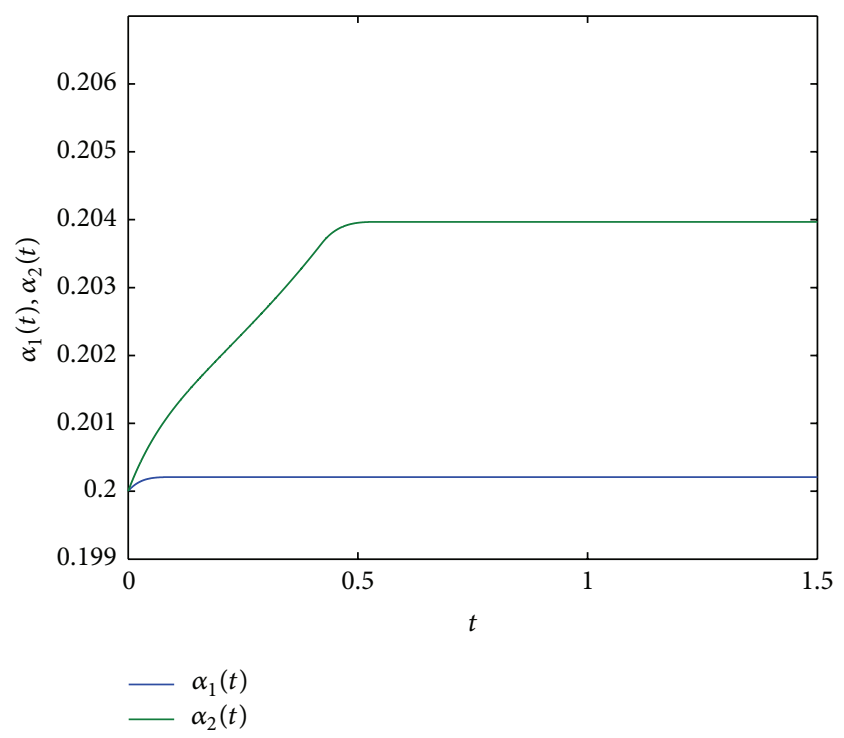

FIGURE 5: Trajectories of control parameters $\alpha_{1}(t)$ and $\alpha_{2}(t)$.

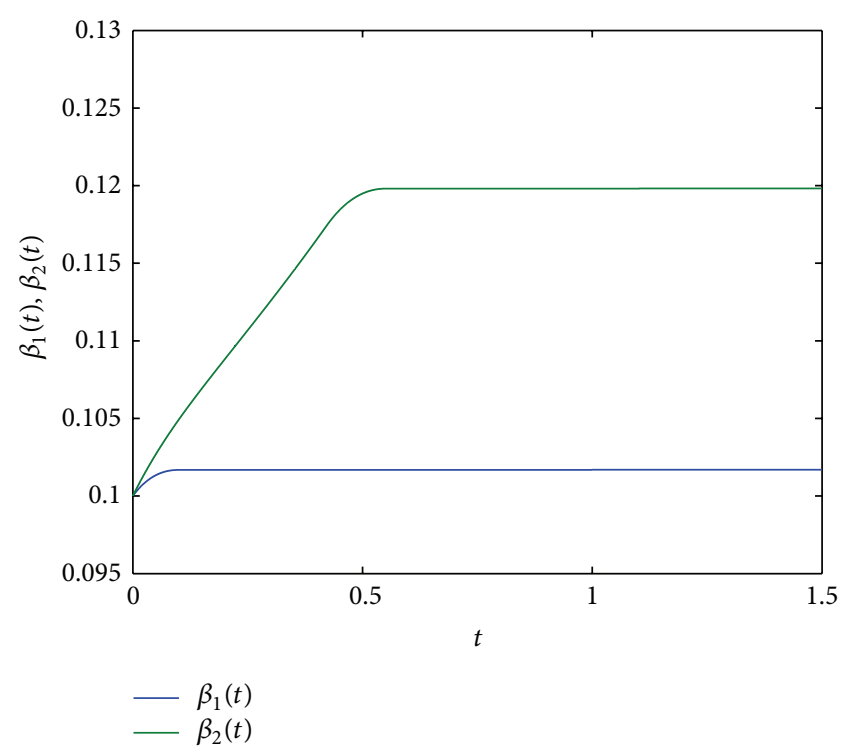

FIGURE 6: Trajectories of control parameters $\beta_{1}(t)$ and $\beta_{2}(t)$.

existence of periodic solution of a class of memristor-based recurrent neural network is also much different from the existing work. The obtained results are also applicable to the continuous systems without switching jumps. Finally, a numerical example has been given to illustrate the validity of the present results.

\section{Acknowledgments}

This work was supported by the Natural Science Foundation of Hebei Province of China (A2011203103) and the Hebei Province Education Foundation of China (2009157). 


\section{References}

[1] L. Chua, "Memristor-the missing circut element," IEEE Transactions on Circuit Theory, vol. 18, pp. 507-519, 1971.

[2] D. Strukov, G. Snider, G. Stewart, and R. Williams, "The missing memristor found," Nature, vol. 453, pp. 80-83, 2008.

[3] J. Tour and T. He, "The fourth element," Nature, vol. 453, pp. 42-43, 2008.

[4] M. Itoh and L. O. Chua, "Memristor oscillators," International Journal of Bifurcation and Chaos in Applied Sciences and Engineering, vol. 18, no. 11, pp. 3183-3206, 2008.

[5] X. Wang, Y. Chen, Y. Gu, and H. Li, "Spintronic memristor temperature sensor," IEEE Electron Device Letters, vol. 31, pp. 20-22, 2010.

[6] R. Riaza, "Nondegeneracy conditions for active memristive circuits," IEEE Transactions on Circuits and Systems II, vol. 57, pp. 223-227, 2010.

[7] F. Merrikh-Bayat and S. Shouraki, "Memristor-based circuits for performing basic arithmetic operations," Procedia Computer Science, vol. 3, pp. 128-132, 2011.

[8] F. Merrikh-Bayat and S. Shouraki, "Programming of memristor crossbars by using genetic algorithm," Procedia Computer Science, vol. 3, pp. 232-237, 2011.

[9] F. Corinto, A. Ascoli, and M. Gilli, "Nonlinear dynamics of memristor oscillators," IEEE Transactions on Circuits and Systems. I, vol. 58, no. 6, pp. 1323-1336, 2011.

[10] A. Wu and Z. Zeng, "Exponential stabilization of memristive neural networks with time delays," IEEE Transactions on Neural Networks and Learning Systems, vol. 23, pp. 1919-1929, 2012.

[11] A. Wu, S. Wen, Z. Zeng, X. Zhu, and J. Zhang, "Exponential synchronization of memristor-based recurrent neural networks with time delays," Neurocomputing, vol. 74, pp. 3043-3050, 2011.

[12] $\mathrm{A}$. Wu and Z. Zeng, "Synchronization control of a class of memristor-based recurrent neural networks," Information Sciences, vol. 183, pp. 106-116, 2012.

[13] A. Wu and Z. Zeng, "Anti-synchronization control of a class of memristive recurrent neural networks," Communications in Nonlinear Science and Numerical Simulation, vol. 18, no. 2, pp. 373-385, 2013.

[14] S. Wen, Z. Zeng, and T. Huang, "Adaptive synchronization of memristor-based Chua's circuits," Physics Letters A, vol. 376, pp. 2775-2780, 2012.

[15] S. Wen and Z. Zeng, "Dynamics analysis of a class of memristorbased recurrent networks with time-varying delays in the presence of strong external stimuli," Neural Processing Letters, vol. 35, pp. 47-59, 2012.

[16] A. Wu and Z. Zeng, "Dynamic behaviors of memristor-based recurrent neural networks with time-varying delays," Neural Networks, vol. 36, pp. 1-10, 2012.

[17] S. Wen, Z. Zeng, and T. Huang, "Exponential stability analysis of memristor-based recurrent neural networks with time-varying delays," Neurocomputing, vol. 97, pp. 233-240, 2012.

[18] G. Zhang, Y. Shen, and J. Sun, "Global exponential stability of a class of memristor-based recurrent neural networks with timevarying delays," Neurocomputing, vol. 97, pp. 149-154, 2012.

[19] A. Wu, J. Zhang, and Z. Zeng, "Dynamic behaviors of a class of memristor-based Hopfield networks," Physics Letters A, vol. 375, no. 15, pp. 1661-1665, 2011.

[20] G. Zhang and Y. Shen, "Global exponential periodicity and stability of a class of memristor-based recurrent neural networks with multiple delays," Information Sciences, vol. 232, pp. 386396, 2013.
[21] S. Wen, Z. Zeng, and T. Huang, "Dynamic behaviors of memristor-based delayed recurrent networks," Neural Computing and Applications, 2012.

[22] X. Liu, J. Cao, and G. Huang, "Complete periodic synchronization of delayed neural networks with discontinuous activations," International Journal of Bifurcation and Chaos in Applied Sciences and Engineering, vol. 20, no. 7, pp. 2151-2164, 2010.

[23] W. Zou and M. Zhan, "Complete synchronization in coupled limit-cycle systems," Europhysics Letters, vol. 81, no. 1, p. 1006, 2008.

[24] W. Zou and M. Zhan, "Complete periodic synchronization in coupled systems," Chaos, vol. 18, no. 4, Article ID 043115, 2008.

[25] J.-P. Aubin and H. Frankowska, Set-Valued Analysis, Birkhäuser, Boston, Mass, USA, 1990.

[26] A. Filippov, Differential Equations With Discontinuous RightHand Side, Mathematics and Its Applications, Soviet, Kluwer Academic, Boston, Mass, USA, 1984.

[27] M. Forti and P. Nistri, "Global convergence of neural networks with discontinuous neuron activations," IEEE Transactions on Circuits and Systems. I. Fundamental Theory and Applications, vol. 50, no. 11, pp. 1421-1435, 2003.

[28] Z. Cai, L. Huang, Z. Guo, and X. Chen, "On the periodic dynamics of a class of time-varying delayed neural networks via differential inclusions," Neural Networks, vol. 33, pp. 97-113, 2012.

[29] H. Wu and C. Shan, "Stability analysis for periodic solution of BAM neural networks with discontinuous neuron activations and impulses," Applied Mathematical Modelling, vol. 33, no. 6, pp. 2564-2574, 2009.

[30] H. Wu, "Global stability analysis of a general class of discontinuous neural networks with linear growth activation functions," Information Sciences, vol. 179, no. 19, pp. 3432-3441, 2009.

[31] A. Berman and R. J. Plemmons, Nonnegative Matrices in the Mathematical Sciences, Academic Press, New York, NY, USA, 1979.

[32] Y. Li and Z. H. Lin, "Periodic solutions of differential inclusions," Nonlinear Analysis. Theory, Methods \& Applications, vol. 24, no. 5, pp. 631-641, 1995. 


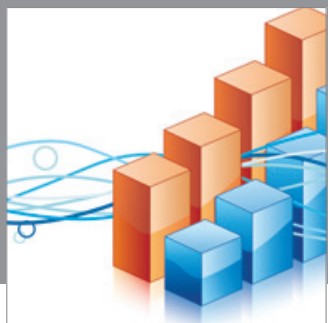

Advances in

Operations Research

mansans

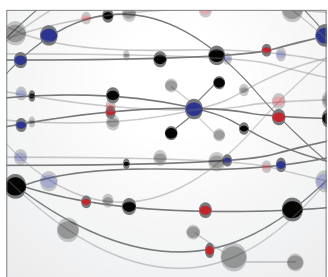

The Scientific World Journal
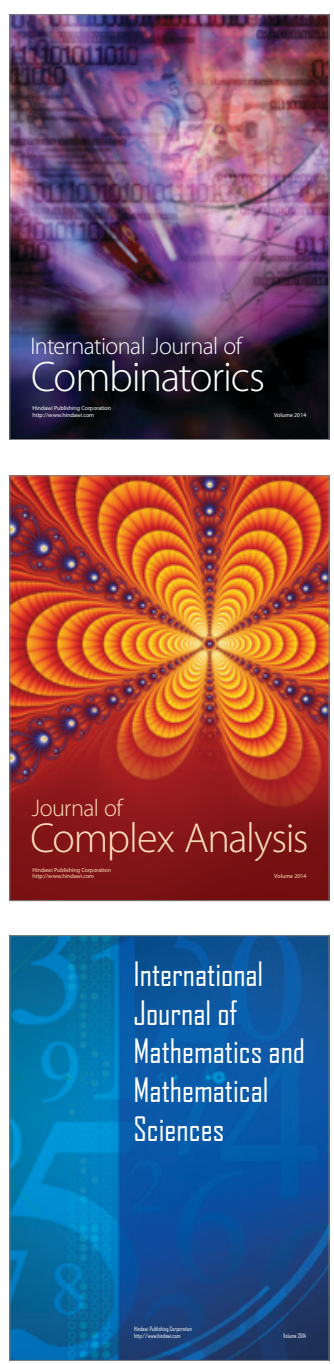
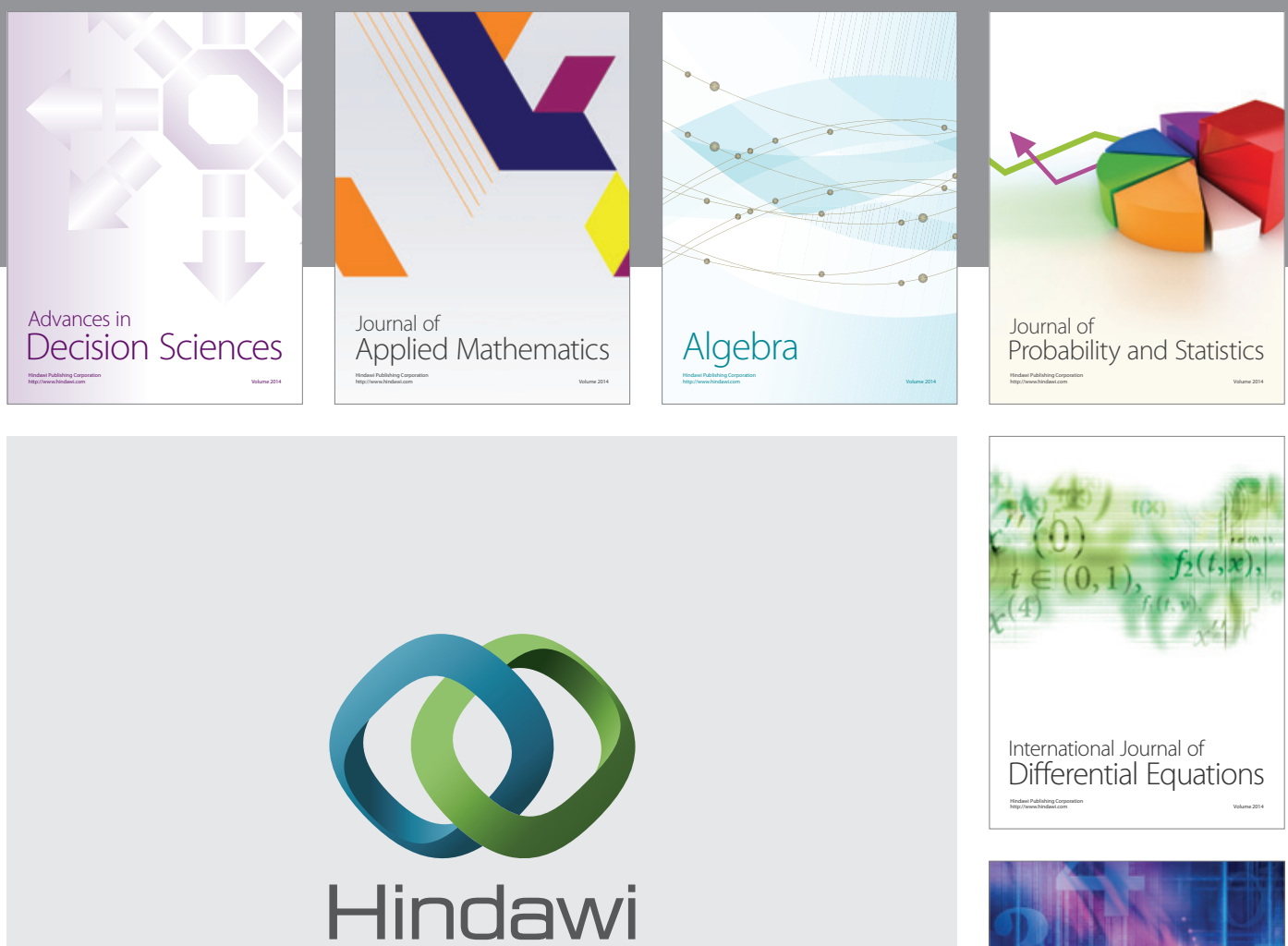

Submit your manuscripts at http://www.hindawi.com
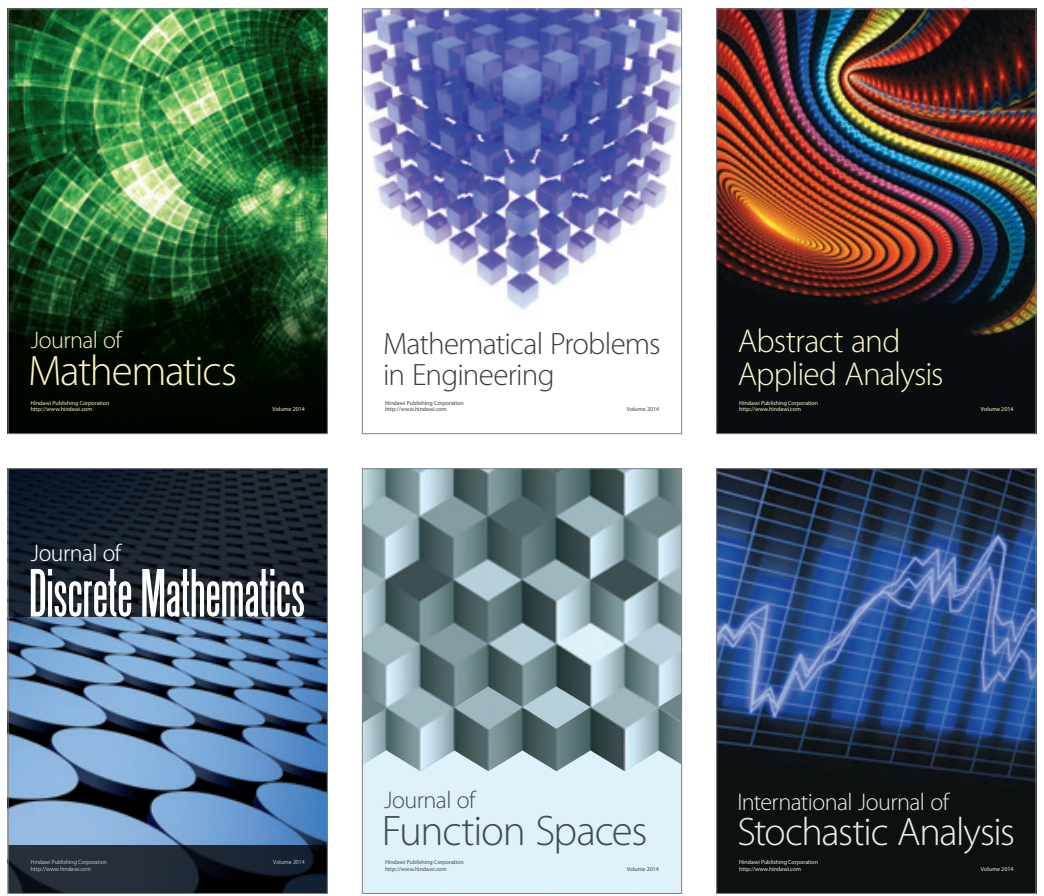

Journal of

Function Spaces

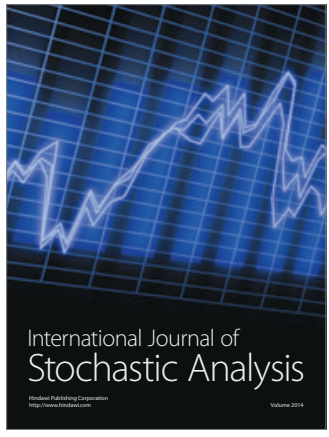

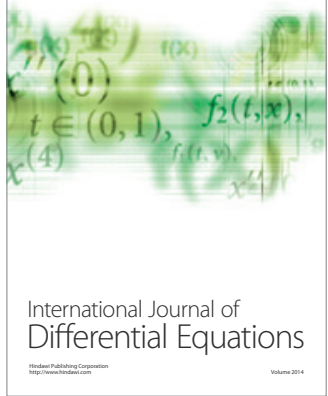
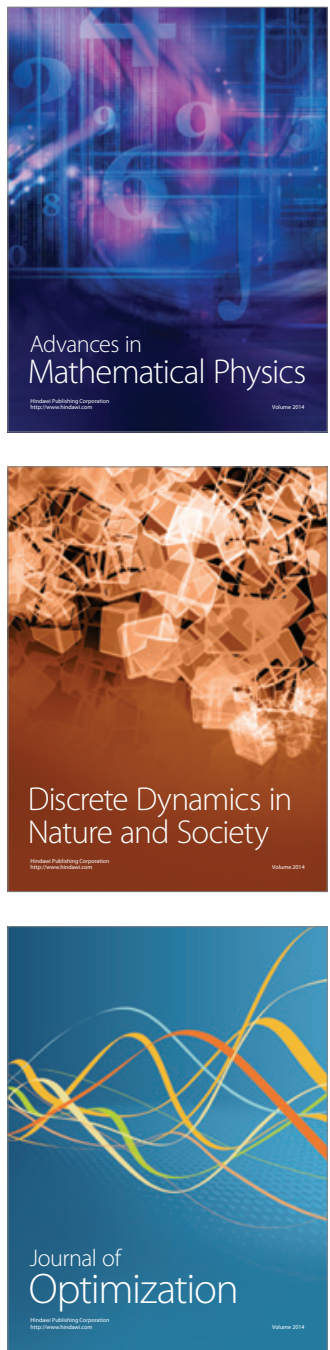